Pré-Publicações do Departamento de Matemática

Universidade de Coimbra

Preprint Number 07-05

\title{
IDEALS IN HEYTING SEMILATTICES AND OPEN HOMOMORPHISMS
}

\author{
JORGE PICADO, ALEŠ PULTR AND ANNA TOZZI
}

\begin{abstract}
Subfitness and its relation to openness and completeness is studied in the context of Heyting semilattices. A formally weaker condition (c-subfitness) is shown to be necessary and sufficient for openness and completeness to coincide. For a large class of spatial frames, c-subfit $\equiv$ subfit.
\end{abstract}

KEYWORDs: Heyting semilattice, ideal, subfitness, open and complete ideals and homomorphisms.

AMS SubJect Classification (2000): 03G25, 06A12, 06D20, 06D22, 54C10.

\section{Introduction}

Recall that a Heyting semilattice (also implicative semilattice [11], or Brouwerian semilattice [10]) is a (meet-)semilattice with the (Heyting) operation $\rightarrow$ satisfying

$$
a \wedge b \leq c \quad \text { iff } \quad a \leq b \rightarrow c .
$$

We study ideals and complete ideals (see 2.2 below) in these objects, show that some standard facts about subfitness (an important concept of topology and logic) hold in this general context, and discuss the question of openness confronted with completeness.

One of the motivations comes from modelling open continuous maps in point-free topology. The condition that images of open sublocales under a frame homomorphism $h: L \rightarrow M$ are open reduces to the existence of a map $\phi: M \rightarrow L$ such that

$$
x \wedge \phi(a)=y \wedge \phi(a) \quad \text { iff } \quad h(x) \wedge a=h(y) \wedge a .
$$

From this equivalence one can easily deduce the famous theorem by Joyal and Tierney ([9]) stating that

$$
h: L \rightarrow M \text { is open iff it is a complete Heyting homomorphism, }
$$

Received January 22, 2007.

The first author gratefully acknowledges partial financial support by Centro de Matemática da Universidade de Coimbra/FCT.

The second author would like to express his thanks for support by project 1M0021620808 of the Ministry of Education of the Czech Republic and to the Italian grant PRIN 2004010554-002. 
that is, if it preserves all joins, all meets and, moreover, also the Heyting operation (always existing due to the frame distributivity).

The condition (Open) can be viewed as a comparison of two frame congruences

$$
E=\{(x, y) \mid x \wedge \phi(a)=y \wedge \phi(a)\} \text { and } F=\{(x, y) \mid h(x) \wedge a=h(y) \wedge a\} .
$$

Now under a certain condition (called fitness, weaker then the point-free variant of regularity), frame congruences coincide iff the classes of the top element do, and (Open) further reduces to

$$
x \wedge \phi(a)=\phi(a) \quad \text { iff } \quad h(x) \wedge a=a,
$$

that is,

$$
\phi(a) \leq x \quad \text { iff } \quad a \leq h(x)
$$

Hence, $h$ is here open iff it is complete, the Heyting part being automatic.

In fact, fitness is not really necessary, and the question naturally arises how far it can be relaxed. It turns out that for every complete homomorphism $h: L \rightarrow M$ being Heyting, it suffices that $L$ is subfit (in spaces, a condition weaker than $T_{1}$ ). We prove a necessary and sufficient condition (formally weaker than subfitness, but a dividing example is still lacking).

Working in the context of Heyting semilattices makes the results substantially more general. The point is, however, not in generalizing for generalization sake (although even this has its merits, making several facts more transparent). Our main aim is, rather, to prove as much as possible without using infinite joins or meets (completeness, for instance, is expressed by the existence of a Galois adjoint, not by preserving arbitrary meets - which do not have to exist at all).

The paper is divided into five sections. After the necessary preliminaries (Section 1) we study, in Section 2, the ideals in Heyting semilattices, the central notion of our investigation. Section 3 is devoted to subfitness and a formally weaker c-subfitness (the necessary and sufficient condition mentioned above); the results are then applied in Section 5 to frames (here we also show that for $T_{D}$-spaces the two conditions coincide, and subfitness is hence necessary and sufficient for every complete homomorphism being open). 


\section{Preliminaries}

1.1. We use the standard notions and notation for posets (partially ordered sets) as e.g. in [3]. We write

$$
\uparrow M=\{x \in X \mid x \geq m \in M\}
$$

and similarly $\downarrow M$ for $M \subseteq X=(X, \leq)$. The least (resp. largest) element, if it exists, will be usually denoted by 0 (resp. 1).

Monotone maps $f: X \rightarrow Y$ and $g: Y \rightarrow X$ are (Galois) adjoint ( $f$ on the left, $g$ on the right) if

$$
f(x) \leq y \quad \text { iff } \quad x \leq g(y) .
$$

It is a well-known fact that

(*) left (resp. right) adjoints preserve all the existing suprema (resp. infima).

1.2. A (meet-) semilattice is a poset $L=(X, \leq)$ such that every couple $\{x, y\} \subseteq X$ has an infimum, usually called meet and denoted by $x \wedge y$. If all the $\{x, y\} \subseteq X$ also have suprema these will be denoted by $x \vee y$ and referred to as joins; $L$ is then said to be a lattice.

1.2.1. Convention. We use the symbol $\sup \{x, y\}$ (as opposed to $x \vee y$ ) in the cases where the suprema do not have to exist. Thus, $" \sup \{a, b\}=c "$ states that $\sup \{a, b\}$ exists and is equal to $c$; or, if 1 exists, $" \sup \{a, b\} \neq 1$ " states that $a$ and $b$ have a common upper bound $c<1$.

1.2.2. If there are suprema and infima of all subsets one speaks of a complete lattice. Here one has the converse of the $(*)$ above, namely

a monotone map between complete lattices preserving all the suprema (resp. infima) is a left (resp. right) adjoint.

1.3. A pseudocomplement of an element $a$ of a semilattice $L$ with a least element is an $a^{*} \in L$ such that

$$
x \wedge a=0 \quad \text { iff } \quad x \leq a^{*} .
$$

1.4. A non-empty semilattice is a Heyting semilattice if there is a binary operation $\rightarrow$ satisfying

$$
a \wedge b \leq c \quad \text { iff } \quad a \leq b \rightarrow c .
$$


Note that

if a Heyting semilattice has a least element 0, it has pseudocomplements, namely $a^{*}=a \rightarrow 0$.

Lattices with an operation $\rightarrow$ satisfying $(\mathrm{H})$ are called Heyting algebras.

1.5. A few Heyting formulas. In the sequel, the use of $(H)$ is mostly automatic.

Proposition. In a Heyting semilattice $H$ we have:

(1) $a \leq b \rightarrow c \quad$ iff $\quad b \leq a \rightarrow c$,

(2) $a \rightarrow(b \wedge c)=(a \rightarrow b) \wedge(a \rightarrow c)$,

(3) there is a largest element 1 and $a \rightarrow a=1$ for all $a$,

(4) $a \leq b$ iff $a \rightarrow b=1$,

(5) $a \leq b \rightarrow a$,

(6) $a \rightarrow b=a \rightarrow(a \wedge b)$,

(7) $a \wedge(a \rightarrow b)=a \wedge b$,

(8) $a \wedge b=a \wedge c \quad$ iff $a \rightarrow b=a \rightarrow c$,

(9) $(a \wedge b) \rightarrow c=a \rightarrow(b \rightarrow c)$.

If $H$ is a Heyting algebra we have, furthermore, that

(10) $H$ is a distributive lattice, and

(11) for every $a, b \in H, \quad b=(b \vee a) \wedge(a \rightarrow b)$.

Proof. (1) follows immediately from $(\mathrm{H})$ and the commutativity of $\wedge$.

(2) since $a \rightarrow(-)$ is a right adjoint (recall 1.1).

(3) $x \leq a \rightarrow a$ iff $x \wedge a \leq a$, that is, always.

(4) $1 \leq a \rightarrow b$ iff $a=1 \wedge a \leq b$.

(5) since $a \wedge b \leq a$.

(6) by (2) and (3).

(7) $a \wedge(a \rightarrow b) \leq b$ since $a \rightarrow b \leq a \rightarrow b$, and $a \wedge b \leq a \wedge(a \rightarrow b)$ by (5).

(8) follows from (2) and (7).

(9) $x \leq(a \wedge b) \rightarrow c$ iff $x \wedge a \wedge b \leq c$ iff $x \wedge a \leq b \rightarrow c$ iff $x \leq a \rightarrow(b \rightarrow c)$.

(10) $(-) \wedge a$ is a left adjoint and hence preserves all the existing suprema.

$(11) b \leq(b \vee a) \wedge(a \rightarrow b)$ by $(5)$; by $(10)$ and $(7),(b \vee a) \wedge(a \rightarrow b)=(b \wedge(a \rightarrow$ b)) $\vee(a \wedge(a \rightarrow b)) \leq b$. 


\section{Ideals in Heyting semilattices}

2.1. There are two main reasons for working with Heyting semilattices. First, the central notion of ideal fits to this structure rather than to Heyting algebras. Second, in the facts about subfitness (Section 3, applied in Section $5)$ the join does not play any role. Some specific facts concerning Heyting algebras are discussed in Section 4.

The system of Heyting semilattices can be viewed as a variety of algebras (if we use the suggestive + for $\wedge$ and $\cdot$, or just a juxtaposition, for $\rightarrow$, it can be determined by the equations

$$
\begin{aligned}
& a+(b+c)=(a+b)+c, \quad a+b=b+a, \quad a+a=a, \\
& a(b+c)=a b+a c, \quad a+b(a+b)=a, \quad a+a b=a+b
\end{aligned}
$$

since one of the equations is the one-sided distributivity law the definition of ideal below is natural: it is a non-void subset $S \subseteq H$ closed under + and such that for $s \in S$ and any $a \in H$, as $\in S$ ).

2.2. A non-void subset $S$ of a Heyting semilattice $H$ is an ideal if

(I1) $s, t \in S \Rightarrow s \wedge t \in S$, and

(I2) $a \in H \quad \& \quad s \in S \Rightarrow a \rightarrow s \in S$.

We speak of a strong ideal if, moreover,

(Istr) the embedding $j_{S}: S \subseteq H$ is a right adjoint, that is, there is a mapping $\nu_{S}: H \rightarrow S$ such that

$$
\begin{aligned}
& \forall a \in H, s \in S, \quad \nu_{S}(a) \leq s \text { iff } a \leq s \\
& \text { (in other words, } \nu_{S} j_{S}=\mathrm{id}, \text { and } j_{S} \nu_{S} \geq \mathrm{id} \text { ). }
\end{aligned}
$$

A complete ideal has, furthermore, a left adjoint $\phi_{S}$ to $\nu_{S}$ (hence $\nu_{S}$ is both a right and a left adjoint), that is

(Icpl) there is a mapping $\phi_{S}: S \rightarrow H$ such that

$$
\begin{aligned}
& \forall a \in H, s \in S, \quad \phi_{S}(s) \leq a \text { iff } s \leq \nu_{S}(a) \\
& \text { (in other words, } \nu_{S} \phi_{S} \leq \mathrm{id}, \text { and } \phi_{S} \nu_{S}=\mathrm{id} \text { ). }
\end{aligned}
$$

2.3. Observations. (1) Each ideal contains the top 1 (indeed, let $s \in S$; then $1=s \rightarrow s \in S$ ).

(2) Ideals in $H$ are Heyting sub-semilattices of $H$. 
2.4. Obviously, the intersection of any system $S_{i}, i \in J$, of ideals is an ideal. The complete lattice of ideals of $H$ will be denoted by

$$
\operatorname{ldl}(H) \text {. }
$$

Note that the least element in this lattice is $O=\{1\}$.

2.4.1. Proposition. The join of two ideals in $\operatorname{ldl}(H)$ is given by

$$
S \vee T=\{s \wedge t \mid s \in S, t \in T\} .
$$

Proof. Obviously any ideal $U$ containing $S, T$ contains $S \vee T$ which is an ideal $($ as $a \rightarrow(s \wedge t)=(a \rightarrow s) \wedge(a \rightarrow t)$ by 1.5(2)).

2.4.2. Proposition. If $S, T$ are strong ideals then $S \vee T$ is strong. Thus, the system

$$
\widetilde{\mathrm{d}} \mathrm{l}(H)
$$

of strong ideals is a sub-join-semilattice of $\operatorname{Idl}(H)$.

Proof. Set $\nu(x)=\nu_{S}(x) \wedge \nu_{T}(x)$. Thus, for a general $x, x \leq \nu(x)$, and for $s \wedge t(s \in S, t \in T)$ we have

$$
\nu(s \wedge t)=\nu_{S}(s \wedge t) \wedge \nu_{T}(s \wedge t)=s \wedge \nu_{S}(t) \wedge \nu_{T}(s) \wedge t=s \wedge t .
$$

2.5. Proposition. $\operatorname{Idl}(H)$ is a distributive lattice.

Proof. Let $S_{1}, S_{2}, T$ be ideals. Then trivially $\left(S_{1} \cap S_{2}\right) \vee T \subseteq\left(S_{1} \vee T\right) \cap\left(S_{2} \vee T\right)$. Now let $x \in\left(S_{1} \vee T\right) \cap\left(S_{2} \vee T\right)$. Then $x=s_{1} \wedge t_{1}=s_{2} \wedge t_{2}$ with $s_{i} \in S_{i}$ and $t_{1}, t_{2} \in T$. Set $t=t_{1} \wedge t_{2}$. Then $x=\left(s_{1} \wedge t_{1}\right) \wedge\left(s_{2} \wedge t_{2}\right) \leq s_{i} \wedge t \leq x$ and we have $s_{1} \wedge t=x=s_{2} \wedge t$, and by 1.5(8) $t \rightarrow s_{1}=t \rightarrow s_{2}$. Thus $t \rightarrow s_{1} \in S_{1} \cap S_{2}$ and we have $x=\left(t \rightarrow s_{1}\right) \wedge t \in\left(S_{1} \cap S_{2}\right) \vee T$.

Note. It has been pointed out to us by P.T. Johnstone that the first short proof of the distributivity in similar vein, for the case of sublocales in frames, is due to Dana Scott — see also [8].

2.6. Proposition. Let $S, T$ be ideals (strong ideals, complete ideals, resp.) in $H$ and let $S \subseteq T$. Then $S$ is an ideal (strong ideal, complete ideal, resp.) in $T$.

Proof. The statement for ideals is straightforward. Now for the strong case, let $j_{S}: S \subseteq H, j^{\prime}: S \subseteq T$ and $j_{T}: T \subseteq H$ be the embeddings and let $\nu_{S}, \nu_{T}$ be the adjoints. Thus,

$$
\nu_{S} j_{S}=\mathrm{id}, \nu_{T} j_{T}=\mathrm{id}, j_{S} \nu_{S} \geq \mathrm{id} \text { and } j_{T} \nu_{T} \geq \mathrm{id} .
$$


Set $\nu^{\prime}=\nu_{S} j_{T}$. Then $\nu^{\prime} j^{\prime}=\nu_{S} j_{T} j^{\prime}=\nu_{S} j_{S}=\mathrm{id}$, and $j_{T} j^{\prime} \nu^{\prime}=j_{S} \nu_{S} j_{T} \geq \mathrm{id} \cdot j_{T}$ and hence (as $j_{T}$ is an order embedding), $j^{\prime} \nu^{\prime} \geq \mathrm{id}$.

For the complete case consider the left adjoints $\phi_{S}, \phi_{T}$ to $\nu_{S}, \nu_{T}$. We have

$$
\nu_{S} \phi_{S}=\mathrm{id}, \nu_{T} \phi_{T}=\mathrm{id}, \phi_{S} \nu_{S} \leq \mathrm{id} \text { and } \phi_{T} \nu_{T} \leq \mathrm{id} .
$$

Set $\phi^{\prime}=\nu_{T} \phi_{S}$. Then $\phi^{\prime} \nu^{\prime}=\nu_{T} \phi_{S} \nu_{S} j_{T} \leq \nu_{T} j_{T}=$ id and

$$
\nu^{\prime} \phi^{\prime}=\nu_{S} j_{T} \nu_{T} \phi_{S}=\nu^{\prime} \nu_{T} j_{T} \nu_{T} \phi_{S}=\nu^{\prime} \nu_{T} \phi_{S}=\nu_{S} \phi_{S}=\mathrm{id}
$$

$\left(\nu^{\prime} \nu_{T}=\nu_{S}\right.$ since $j_{T} j^{\prime}=j_{S}$, and $\nu_{T} j_{T} \nu_{T}=\nu_{T}$ is standard $)$.

2.7. Open (principal) ideals. The operation $\rightarrow$ distributes over meets on the left, and $y \rightarrow(a \rightarrow x)=a \rightarrow(y \rightarrow x)$ (recall 1.5(9)). Thus, we have the principal ideals in $H$

$$
\mathfrak{o}(a)=\{a \rightarrow x \mid x \in H\}
$$

(in the $(+, \cdot)$-notation of 2.1 of the distributivity $a(x+y)=a x+a y$ and the equation $y(a x)=a(y x)$ we have $\mathfrak{o}(a)=\{a x \mid x \in H\})$. Because of their role in pointfree topology we speak of them as of open ideals. Observe that

$$
\mathfrak{o}(a)=\{x \mid a \rightarrow x=x\}
$$

(use 1.5(9)). Each $\mathfrak{o}(a)$ is a complete ideal. It is strong $\left(\right.$ set $\nu_{\mathfrak{o}(a)}(x)=a \rightarrow x$; then $\nu j(x)=a \rightarrow x=x$ and $j \nu(y)=a \rightarrow y \geq y)$, and we have $\phi_{\mathfrak{o}(a)}(x)=a \wedge x$ adjoint to $\nu_{\mathfrak{o}(a)}$.

2.7.1. Proposition. (1) $a \leq b$ iff $\mathfrak{o}(a) \subseteq \mathfrak{o}(b)$.

(2) $\mathfrak{o}(a \wedge b)=\mathfrak{o}(a) \cap \mathfrak{o}(b)$.

Proof. (1.5(9) used repeatedly)

(1) If $a \leq b$ and $x=a \rightarrow y \in \mathfrak{o}(a)$ we have

$$
b \rightarrow x=b \rightarrow(a \rightarrow y)=(b \wedge a) \rightarrow y=a \rightarrow y=x \in \mathfrak{o}(b) .
$$

Let $\mathfrak{o}(a) \subseteq \mathfrak{o}(b)$ then $a \rightarrow(a \wedge b)$ is in $\mathfrak{o}(b)$ and hence

$$
a \rightarrow(a \wedge b)=b \rightarrow(a \rightarrow(a \wedge b))=(a \wedge b) \rightarrow(a \wedge b)=1
$$

so that $a \leq a \wedge b$ and finally $a \leq b$.

(2) Trivially $\mathfrak{o}(a) \cap \mathfrak{o}(b) \supseteq \mathfrak{o}(a \wedge b)$. Now let $x \in \mathfrak{o}(a) \cap \mathfrak{o}(b)$. Then $x=a \rightarrow$ $x=b \rightarrow x$ and hence $(a \wedge b) \rightarrow x=a \rightarrow(b \rightarrow x)=a \rightarrow x=x$.

2.7.2. Proposition. For any open ideal $\mathfrak{o}_{S}(a)$ in an ideal $S \subseteq H$ we have $\mathfrak{o}_{S}(a)=\mathfrak{o}(a) \cap S$. Consequently, an open ideal in an open ideal in $H$ is itself an open ideal in $H$. 
Proof. The first is obvious: we have $\mathfrak{o}_{S}(a)=\{x \mid x \in S, a \rightarrow x=x\}$. Now in particular $\mathfrak{o}_{\mathfrak{o}(b)}(a)=\mathfrak{o}(a) \cap \mathfrak{o}(b)=\mathfrak{o}(a \wedge b)=\mathfrak{o}(a)$, by 2.7.1(2) $(a \wedge b=a$ by $2.7 .1(1))$.

2.8. Proposition. An ideal $S \subseteq H$ is open iff it is complete and if $\nu_{S}$ preserves the Heyting operation.

Proof. Let $S=\mathfrak{o}(a)$ be open. We already know it is complete. Now for $\nu=\nu_{\mathfrak{o}(a)}$ we have

$$
\nu(x \rightarrow y)=a \rightarrow(x \rightarrow y)=(a \wedge x) \rightarrow y=(a \wedge(a \rightarrow x)) \rightarrow y
$$

(by $1.5(7)$ and $(9)$ ) and proceed, by $1.5(9), \ldots=(a \rightarrow x) \rightarrow(a \rightarrow y)=\nu(x) \rightarrow$ $\nu(y)$.

Conversely, let $S$ be complete and let $\nu=\nu_{S}$ preserve the Heyting operation. Set $a=\phi_{S}(1)$. Then we have

$$
\nu_{S}(a \rightarrow x)=\nu(\phi(1)) \rightarrow \nu(x)=1 \rightarrow \nu(x)=\nu(x)
$$

and hence $a \rightarrow x \leq \nu(x)$. On the other hand,

$$
1=\nu(x) \rightarrow \nu(x)=\nu(\nu(x)) \rightarrow \nu(x)=\nu(\nu(x) \rightarrow x)
$$

and hence $a=\phi(1) \leq \nu(x) \rightarrow x, a \wedge \nu(x) \leq x$, and finally $\nu(x) \leq a \rightarrow x$, and hence $\nu(x)=a \rightarrow x$, and $S=\nu[H]=\{a \rightarrow x \mid x \in H\}=\mathfrak{o}(a)$.

2.8.1. Corollary. Principal ideals are retracts in the category of Heyting semilattices.

2.9. Closed ideals. Those are

$$
\mathfrak{c}(a)=\uparrow a=\{x \mid x \geq a\} .
$$

$\mathfrak{c}(x)$ is indeed an ideal : meet is trivial and if $x \geq a$ then (by 1.5(5)) $y \rightarrow x \geq$ $x \geq a$. In the general case it is not strong, but see 4.2 below.

2.10. For an ideal $S$ set

$$
\mathfrak{n}(S)=\{x \in H \mid x \leq s \in S \Rightarrow s=1\}, \quad \partial S=\downarrow(S \backslash\{1\}) .
$$

2.10.1. Observations. (1) In the strong case, $\mathfrak{n}(S)=\nu_{S}^{-1}(1)$.

(2) For closed ideals, $\mathfrak{n}(\mathfrak{c}(a))=\{x \mid \sup \{a, x\}=1\}$.

2.10.2. Lemma. If $a \in \mathfrak{n}(S)$ then for every $s \in S, a \rightarrow s=s$.

Proof. As $a \wedge(a \rightarrow s) \leq s$, we have $a \leq(a \rightarrow s) \rightarrow s$, hence $(a \rightarrow s) \rightarrow s=1$ and $a \rightarrow s \leq s$, while $s \leq a \rightarrow s$ is trivial. 
2.10.3. Proposition. The following statements are equivalent:

(1) $S \subseteq \mathfrak{o}(a)$,

(2) $\partial S \subseteq \partial \mathfrak{o}(a)$

(3) $a \in \mathfrak{n}(S)$ (in the strong case, $\nu_{S}(a)=1$ ),

(4) $\mathfrak{c}(a) \cap S=0$.

Proof. $(1) \Rightarrow(2)$ is trivial.

$(2) \Rightarrow(3)$ : Let $a \leq s \in S$. Suppose $s \notin \mathfrak{n}(S)$, that is, $s \in \partial S$. Then $s \in \partial \mathfrak{o}(a)$ and there is an $x<1$ such that $s \leq x=a \rightarrow x$, hence $a=s \wedge a \leq x$, and $x=a \rightarrow x=1$, a contradiction. For the statement on the $\nu$ see Observation 2.10.1.

$(3) \Leftrightarrow(4)$ is just a reformulation.

$(3) \Rightarrow(1)$ follows from Lemma 2.10.2.

2.11. Comparing (1) and (4) in Proposition 2.10 .3 we immediately obtain Corollary. $\mathfrak{o}(a)$ is the pseudocomplement of $\mathfrak{c}(a)$ in $\operatorname{ldl}(A)$.

\subsection{Properties of $\nu_{S}$.}

(1) For every $x \in H$ and $s \in S, x \rightarrow s=\nu_{S}(x) \rightarrow s$.

(2) $x \leq \nu_{S}(x), \nu_{S}\left(\nu_{S}(x)\right)=\nu_{S}(x)$.

(3) $\nu_{S}(x \wedge y)=\nu_{S}(x) \wedge \nu_{S}(y)$.

Proof. (1) $y \leq x \rightarrow s$ iff $x \leq y \rightarrow s$ iff (by (I2)) $\nu_{S}(x) \leq y \rightarrow s$ iff $y \leq \nu_{S}(x) \rightarrow s$.

(2) is in condition (Istr) in 2.2 .

(3) $x \wedge y \leq \nu(x \wedge y)$, hence $x \leq y \rightarrow \nu(x \wedge y)$, hence $\nu(x) \leq y \rightarrow \nu(x \wedge y)$, hence $y \leq \nu(x) \rightarrow \nu(x \wedge y), \nu(y) \leq \nu(x) \rightarrow \nu(x \wedge y)$ and finally $\nu(x) \wedge \nu(y) \leq \nu(x \wedge y)$. The other inequality is trivial.

Note. The properties in (2) and (3) are the properties of a nucleus as considered in the case of complete Heyting algebras (frames).

\section{Subfit Heyting semilattices}

3.1. A Heyting semilattice is subfit (cf. [6], conjunctive in [14],[15]) if we have the implication

$$
a \not \leq b \quad \Rightarrow \quad \exists c, \sup (a, c)=1 \neq \sup (b, c) .
$$

(Recall 1.2.1: $" \sup (a, c)=1 \neq \sup (b, c) "$ says that $a, c$ do not have a common upper bound in $H$ other than the top while $b, c$ do.) 
3.2. Theorem. Let $H$ be a Heyting semilattice. Then the following statements are equivalent.

(1) $H$ is subfit.

(2) For a strong ideal $S \subseteq H, S \backslash\{1\}$ is cofinal in $H \backslash\{1\}$ only if $S=H$.

(3) If $S$ is a strong ideal such that $S \neq L$ then there is a closed $\mathfrak{c}(x) \neq\{1\}$ such that $S \cap \mathfrak{c}(x)=\{1\}$.

(4) Each open ideal $\mathfrak{o}(a)$ is the supremum in $\operatorname{ldl}(H)$ of the system

$$
\{\mathfrak{c}(x) \mid \sup (x, a)=1\} .
$$

(5) Each open ideal in $H$ is a supremum in $\operatorname{ldl}(H)$ of a system of closed ideals.

Proof. (1) $\Rightarrow(2)$ : Let $b \in H$ and $a=\nu_{S}(b)$. Let $\sup (a, b)=1$ and let $x>1$ be such that $x \geq b, c$. By the cofinality there is an $s \in S, x \leq s<1$. Then $s \geq b$ and being in $S$, it is $\geq a$. Thus $s \geq a, c$, hence $s=1$, a contradiction proving that $b=a \in S$.

$(2) \Rightarrow(3)$ : (3) is just a reformulation of $(2)$.

$(3) \Rightarrow(4)$ : By $2.10 .3, \mathfrak{o}(a)$ is an upper bound of the system. Now let $S$ be a general upper bound; thus, for all $x$ with $\sup (a, x)=1, S \supseteq \mathfrak{c}(x)$. Let $\mathfrak{c}(y) \cap(\mathfrak{c}(a) \vee S)=\{1\}$. Then in particular $\mathfrak{c}(y) \cap \mathfrak{c}(a)=\{1\}$ and hence $\sup (y, a)=1$, by 2.10.3(4), and $\mathfrak{c}(y) \subseteq S$. Thus, $\mathfrak{c}(y)=\mathfrak{c}(y) \cap(\mathfrak{c}(a) \vee S)=\{1\}$ and by $(3), \mathfrak{c}(a) \vee S=H$, and $\mathfrak{o}(a)=\mathfrak{o}(a) \cap(\mathfrak{c}(a) \vee S)=\mathfrak{o}(a) \cap S$, and $\mathfrak{o}(a) \subseteq S$. $(4) \Rightarrow(5)$ is trivial.

$(5) \Rightarrow(1)$ : If $a \not \leq b$ we have $\mathfrak{c}(b) \nsubseteq \mathfrak{c}(a)$ and hence $\mathfrak{o}(a) \nsubseteq \mathfrak{o}(b)$. If $\mathfrak{o}(a)$ is a supremum of a system of closed ideals there is a $c$ such that $\mathfrak{c}(c) \subseteq \mathfrak{o}(a)$, so that $\sup (a, c)=1$, while $\mathfrak{c}(a) \nsubseteq \mathfrak{o}(b)$ and consequently $\sup (c, b) \neq 1$.

3.3. From 2.7 .2 and 3.2.(5) we immediately obtain

Corollary. An open ideal in a subfit Heyting semilattice is itself subfit.

3.4. Proposition. Let $S$ be a complete ideal in a subfit Heyting semilattice. Then $S$ is open.

Thus, completeness yields in the subfit case the automatic preservation of the Heyting operation.

Proof. Set $a=\phi_{S}(1)$. Then, first, $\nu_{S}(a)=\nu_{S}\left(\phi_{S}(1)\right) \geq 1$ and hence, by 2.10.3, $S \subseteq \mathfrak{o}(a)$. 
We will show that $S \backslash\{1\}$ is cofinal in $\mathfrak{o}(a) \backslash 1$. Indeed, if $1 \neq x=a \rightarrow y$ is an element of $\mathfrak{o}(a)$ then $a \not \leq y$, hence $a \wedge a \not \leq y$ and $\phi(1)=a \not \leq a \rightarrow y=x$ so that $1 \not \leq \nu(x)$, that is, $x \leq \nu(x) \neq 1$. Thus, by 3.3 and $3.2(2), S=\mathfrak{o}(a)$.

3.5. Note. In the previous proof, in fact, the subfitness was not used in formally the full strength. It would have sufficed to replace (subfit) by the formally weaker

(c-subfit) for a complete ideal $S \subseteq H, S \backslash\{1\}$ is cofinal in $H \backslash\{1\}$ only if $S=H$.

Or, in other words, by

(c-subfit') for a complete ideal $S \subseteq H, \nu_{S}^{-1}(1)=\{1\}$ only if $S=H$.

We do not know whether this formally weaker condition is really weaker than (subfit). It seems to be likely, but we will show that in an important class of Heyting algebras these two conditions coincide; see 5.3 below.

3.6. Proposition. A Heyting semilattice is subfit (resp. satisfies (c-subfit)) iff for a strong ideal (resp. a complete ideal) $S$ and any open ideal $\mathfrak{o}(a)$

$$
\partial S=\partial \mathfrak{o}(a) \quad \Rightarrow \quad S=\mathfrak{o}(a) .
$$

Proof. $\Leftarrow$ is obvious: apply the formula for $a=1$, that is, $\mathfrak{o}(a)=H$.

$\Rightarrow$ : By 2.10.3, $S \subseteq \mathfrak{o}(a)$. Since $\mathfrak{o}(a)$ is subfit (see 3.3), and since $\partial S=\partial \mathfrak{o}(a)$ makes the subset $S \subseteq \mathfrak{o}(a)$ cofinal, $S=\mathfrak{o}(a)$ by 3.2 .

3.7. Proposition. A Heyting semilattice satisfies (c-subfit) iff every complete ideal in $H$ is open.

Proof. We already know that the condition suffices. Now let each complete ideal be open. By 3.6, to show that it is necessary it suffices to prove that $\partial \mathfrak{o}(a)=\partial \mathfrak{o}(b)$ implies $a=b$. Now if the first holds we have $\nu_{\mathfrak{o}(b)}(x)=1$ iff $\nu_{\mathfrak{o}(a)}=1$, that is, $b \rightarrow x=1$ iff $a \rightarrow x=1$, that is $a \leq x$ iff $b \leq x$.

\section{Intermezzo: The case of Heyting algebras}

4.1. In this section we will discuss Heyting algebras $H$ instead of Heyting semilattices (recall 1.4).

In this case every strong ideal $S$ is again a Heyting algebra, with the binary join being $\nu_{S}(x \vee y)$ and the mapping $\nu_{S}$ a lattice homomorphism. 
If $H$ is a complete Heyting algebra (frame) then the fact that $j_{S}$ has a left adjoint says precisely that $S \subseteq H$ is closed under arbitrary meets, so that the condition (Istr) is in fact an extension of (I1) to all meets. $S$ is then again a frame, with the joins given by $\nu_{S}\left(\bigvee a_{i}\right)$, and $\nu_{S}$ is a frame homomorphism (a sublocale, modelling a generalized subspace if a frame is viewed as a generalized space; the $\mathfrak{o}(a)$ resp. $\mathfrak{c}(a)$ then model the open resp. closed ones).

If $S$ is complete, $\nu_{S}$ is a complete lattice homomorphism. Frame homomorphisms (preserving finite meets and general joins) model continuous maps, and complete Heyting homomorphisms model the open continuous ones (see also 5.1 below).

4.2. Observation. If $H$ is a Heyting algebra then each $\mathfrak{c}(a)$ is a strong ideal, with $\nu_{\mathfrak{c}(a)}(x)=a \vee x$.

4.3. Proposition. If $H$ is a Heyting algebra then $\mathfrak{o}(a)$ and $\mathfrak{c}(a)$ are complements to each other in $\operatorname{ldl}(H)$ (and in $\widetilde{\mathrm{Id}}(H)$ ).

Proof. If $x \in \mathfrak{o}(a) \cap \mathfrak{c}(a)$ then $x \geq a$ and $x=a \rightarrow x=1$. If $x \in H$ is general, then, by 1.5(11), $x=(x \vee a) \wedge(a \rightarrow x) \in \mathfrak{o}(a) \vee \mathfrak{c}(a)$.

4.4. Proposition. Let $S \subseteq H$ be a strong ideal in a Heyting algebra. Then

$$
S=\bigcap\left\{\mathfrak{c}(x) \vee \mathfrak{o}(y) \mid \nu_{S}(x)=\nu_{S}(y)\right\} .
$$

Proof. Let $a \in S$ and let $\nu_{S}(x)=\nu_{S}(y)$. Then

$$
x \rightarrow a=\nu_{S}(x) \rightarrow a=\nu_{S}(y) \rightarrow a=y \rightarrow a
$$

and (recall 1.5(11))

$$
a=(a \vee x) \wedge(x \rightarrow a)=(a \vee x) \wedge(y \rightarrow a) \in \mathfrak{c}(x) \vee \mathfrak{o}(y)
$$

$($ as $y \rightarrow(y \rightarrow a)=y \rightarrow a)$.

Let $a$ be in the intersection. Then for any $x, y$ with $\nu_{S}(x)=\nu_{S}(y), a \in$ $\mathfrak{c}(x) \vee \mathfrak{o}(y)$. In particular, $a \in \mathfrak{c}\left(\nu_{S}(a)\right) \vee \mathfrak{o}(a)$ so that $a=x \wedge(a \rightarrow y)$ for some $x \geq \nu_{S}(a) \geq a$, and $y \in H$. Then by 1.5(3), (2) and (9),

$$
1=a \rightarrow a=(a \rightarrow x) \wedge(a \rightarrow(a \rightarrow y))=1 \wedge(a \rightarrow y)=a \rightarrow y,
$$

and $a=x \geq \nu_{S}(a)$. 
4.5. A Heyting algebra is fit (cf. [6]) if we have the implication

$$
a \not \leq b \quad \Rightarrow \quad \exists c, a \vee c=1, c \rightarrow b \neq b .
$$

4.6. Recall the $\partial S$ from 2.10 .

Theorem. Let $H$ be a Heyting algebra. Then the following statements are equivalent.

(1) $H$ is fit.

(2) For a strong ideal $S$ and an ideal $T$,

$$
\partial T \subseteq \partial S \Rightarrow T \subseteq S .
$$

(3) For each strong ideal $S \subseteq H$,

$$
S=\bigcap\left\{\mathfrak{o}(x) \mid \nu_{S}(x)=1\right\} .
$$

(4) For each closed ideal,

$$
\uparrow a=\bigcap\{\mathfrak{o}(x) \mid a \vee x=1\} .
$$

Remark. Note that in (2) one will not use even the (I1).

Proof. (1) $\Rightarrow(2)$ : Let $\partial T \subseteq \partial S$ and let $b \in T, b \neq 1$. Set $a=\nu_{S}(b)$.

Suppose $a \vee c=1$; if $b \vee c \leq s \in S$ we have $a \leq s$, hence $s \geq a \vee c=1$. Thus, $b \vee c \notin \partial S$ and hence $b \vee c \notin \partial T$. We have, by 1.5(11), $b=(b \vee c) \wedge(c \rightarrow b)$ so that $b \vee c \leq(c \rightarrow b) \rightarrow b \in T$, and $(c \rightarrow b) \rightarrow b=1$. Hence $c \rightarrow b \leq b$, that is, $c \rightarrow b=b$. Thus, $a \leq b$, and $b=\nu_{S}(b) \in S$.

$(2) \Rightarrow(3)$ : Set $T=\bigcap\left\{\mathfrak{o}(x) \mid \nu_{S}(x)=1\right\}$. By 2.10.3, $S \subseteq T$. Now let $a \in \partial T$. Then there is a $t \neq 1, t \in T$ and $a \leq t$. Since $t \in T$ we have $x \rightarrow t=t$ whenever $\nu_{S}(x)=1$. Suppose $a \notin \partial S$. Then $\nu_{S}(a)=1$ and we have $a \rightarrow t=t$. But since $a \leq t$ we have (1.5(4)) $a \rightarrow t=1$ and a contradiction $t=1$. Thus, $\partial T \subseteq \partial S$ and, by $(2), T \subseteq S$.

$(3) \Rightarrow(4)$ is immediate.

(4) $\Rightarrow(1)$ : If $\uparrow a=\bigcup\{\mathfrak{o}(x) \mid a \vee x=1\}$ then if $c \rightarrow b=b$ for all $c$ such that $a \vee c=1$ we obtain $a \leq b$ and $H$ is fit.

4.7. Proposition. Let $H$ be a subfit Heyting algebra and let $S$ be a strong ideal in $H$ that has a complement in $\operatorname{ldl}(H)$. Then $S$ is subfit.

Proof. Obviously if $S_{1}$ is a strong ideal in $S$ and if $S$ is a strong ideal in $H$ then $S_{1}$ is one in $H$. Now let $S_{1} \backslash\{1\}$ be cofinal in $S \backslash\{1\}$. Let $S \vee T=H$ and $S \cap T=\{1\}$. Consider $S_{1} \vee T$. If $1 \neq a \in H$ then in a representation 
$a=s \wedge t, s \in S, t \in T$, either $t \neq 1$ and $a \leq t \in S_{1} \vee T$, or $s \neq 1$ and we have $s_{1} \in S_{1}, s \leq s_{1}<1$, and $a \leq s_{1} \in S_{1} \vee T$. Thus, $S_{1} \vee T \backslash\{1\}$ is cofinal in $H \backslash 1$, hence $S_{1} \vee T=H$ by 3.2 , and $S=S \cap\left(S_{1} \vee T\right)=S \cap S_{1} \subseteq S_{1} \subseteq S$.

4.8. Compare 4.7 with 3.3. Also, recall 3.6 and compare it with " for any two strong ideals $S, T, \partial S=\partial T$ implies $S=T$ " that holds for fit Heyting algebras by 4.6(2). We have here a similar statement for a special $T$. It is natural to ask how special the $T$ has to be. Now, a particular feature of the open ideal $\mathfrak{o}(a)$ is that it is complemented, and hence subfit whenever $H$ is. This may lead to the conjecture that something like the complementarity might be the required special property. But this statement does not hold even for closed ideals.

Indeed, consider a Heyting algebra $H$ that is subfit but not fit, and such that intersections of strong ideals are strong (for instance, the lattice of the open sets of a $T_{1}$-space that is not fit). Then there exists a closed ideal $\uparrow a$ such that

$$
\mathfrak{c}(a)=\uparrow a \neq S=\bigcap\{\mathfrak{o}(a) \mid x \vee a=1\} .
$$

We will prove that, however, $\partial(\uparrow a)=\partial S$, or, in other words, $\nu_{S}(x)=1$ iff $\nu_{\mathfrak{c}(a)}(x)=x \vee a=1$. First, obviously $\nu_{S} \leq \nu_{\mathfrak{c}(a)}$ since $\uparrow a \subseteq S$. Hence if $\nu_{S}(x)=1$ then $x \vee a=1$. On the other hand, let $x \vee c=1$ and let $x \leq t \in S$. Then in particular $s \in \mathfrak{o}(x)$, that is, $x \rightarrow s=s$. As $x \leq s, x \rightarrow s=1$, and $\nu_{S}(x)=1$.

\section{The case of spatial frames}

5.1. Recall 4.1. A typical frame is the lattice $\mathfrak{O}(X)$ of open sets of a topological space $X$, and if $f: X \rightarrow Y$ is a continuous map then $\mathfrak{O}(f)=(U \mapsto$ $\left.f^{-1}(U)\right)$ is a frame homomorphism $\mathfrak{O}(Y) \rightarrow \mathfrak{O}(X)$. Frame homomorphisms give a good representation of continuous maps since for a large class of spaces such $h: \mathfrak{O}(Y) \rightarrow \mathfrak{O}(X)$ are precisely the $\mathfrak{O}(f)$. For more about frames see, e.g., [7] or [12].

Open homomorphisms $h: L \rightarrow M$ between frames are characterized, in among the frame homomorphisms, by the existence of a map $\phi: M \rightarrow L$ such that for all $a \in M$ and $x, y \in L$

$$
x \wedge \phi(a)=y \wedge \phi(a) \quad \text { iff } \quad h(x) \wedge a=h(y) \wedge a,
$$


or equivalently

$$
x \wedge \phi(a) \leq y \quad \text { iff } \quad h(x) \wedge a \leq h(y)
$$

from which, by setting $x=1$ we immediately infer that $\phi$ is a left adjoint to $h$. In general the existence of a left adjoint does not suffice. From (Open') one easily infers

5.1.1. Theorem. (Joyal \& Tierney, [9]) A frame homomorphism $h: L \rightarrow M$ is open iff it is a complete homomorphism preserving the Heyting operation.

(Compare with 2.8.)

5.2. Frame congruences on $L$ (that is, equivalence relations preserving all joins and finite meets) can be equivalently represented by ideals $S \subseteq L$ resp. their nuclei $\nu_{S}$, namely

$$
\nu_{E}(x)=\nu_{S_{E}}(x)=\bigvee\{y \mid y E x\}, \quad S_{E}=\nu_{E}[L] .
$$

If the congruence preserves, furthermore, all meets we speak of a complete congruence.

The open ideals $\mathfrak{o}(a)$ above correspond to the open congruences

$$
x \Delta_{a} y \quad \equiv_{\mathrm{df}} \quad x \wedge a=y \wedge a .
$$

The formula (Open) above says, hence, that

$$
\Delta_{\phi(a)}=\{(x, y) \mid h(x) \wedge a=h(y) \wedge a\}=E .
$$

Thus, requiring that a complete congruence be open is expressed by the implication

$$
\Delta_{\phi(a)} 1=E 1 \quad \Rightarrow \quad \Delta_{\phi(a)}=E .
$$

Applying 3.6 we obtain

5.2.1. Proposition. For a frame $L$, the open homomorphisms coincide with the complete frame homomorphisms iff

(c-subfit) for complete congruences $E$ on $L$,

$$
E 1=1 \Rightarrow E=\{(x, x) \mid x \in L\} .
$$

The condition (subfit) assumes this for any frame congruence. We do not have a dividing example.

5.2.2. Note. Up to isomorphism, if $L$ is a frame, the $\nu_{S}: L \rightarrow S$ are precisely the onto frame homomorphisms. Thus, the condition above characterizes the $L$ for which all the complete onto homomorphisms are open, too. This is explained by the following 
Observation. Each complete one-one frame homomorphism is open (without any condition on the frames involved).

(Indeed, for the left adjoint $\phi$ to be a one-one homomorphism $h: L \rightarrow M$ we have $\phi h=$ id and $h \phi \geq$ id. Consider the condition (Open') in 5.1. If $x \wedge \phi(a) \leq y$ then $h(x) \wedge a \leq h(x) \wedge h(\phi(a)) \leq h(y)$, and if $h(x) \wedge a \leq h(y)$ then $x \wedge \phi(a)=\phi(h(x)) \wedge \phi(a) \leq \phi(h(y))=y$.

5.3. A subset $A$ of a space $X$ is quasiopen (see [5]) if for every open $U$ the set $\uparrow(U \cap A)(\uparrow$ in the specialization order $x \leq y \equiv x \in \overline{\{y\}})$ is open.

5.3.1. Proposition. ([4]) The congruence

$$
U E_{A} V \quad \equiv_{d f} \quad U \cap A=V \cap A
$$

is complete iff $A$ is quasiopen.

5.3.2. Recall that a space $X$ is $T_{D}$ (see, e.g., [1]) if for every $x \in X$ there is an open $U \ni x$ such that $U \backslash\{x\}$ is open. We will need a weaker condition

(*) if there is an $x$ such that $\{x\} \neq \overline{\{x\}}$ then there is an a such that $\{a\} \neq \overline{\{a\}}$ and an open $U \ni a$ such that $U \backslash\{a\}$ is open.

The role of $(*)$ in the following theorem is basically in localizing the fact that in a $T_{D}$ space

$$
E_{A}=E_{B} \Rightarrow A=B([13],[2]) .
$$

Theorem. Let $L=\mathfrak{O}(X)$ and let $X$ satisfy $(*)$. Then the following statements are equivalent.

(1) A frame homomorphism $h: L \rightarrow M$ is open iff it is complete.

(2) $L$ is subfit.

(3) $X$ is $T_{1}$.

Proof. $(3) \Rightarrow(2)$ is trivial and $(2) \Rightarrow(1)$ we already know.

$(1) \Rightarrow(3)$ : Let $X$ not be $T_{1}$; choose $a$ as in $(*)$ and set $A=X \backslash\{a\}$. Then

$A$ is not open, but it is quasiopen: if $U \subseteq X$ is open then $U \cap A=U \backslash\{a\}$ and either $U \backslash\{a\}=U \backslash \overline{\{a\}}$ and it is open itself, or there is an $x$ in $\overline{\{a\}} \cap U \cap A$ and $\uparrow(U \cap A)=U$.

Now if $U E_{A} X$ then $U \backslash\{a\}=X \backslash\{a\}$ and hence $U=X$ (since it contains $X \backslash\{a\}$ and $X \backslash\{a\}$ is not open). Hence $E_{A} X$ is trivial and if (1) holds then the whole of $E_{A}$ is trivial, by 5.2.1. But this is a contradiction since for the $U$ from $(*)$ we have $U E_{A}(U \backslash\{a\})$. 


\section{References}

[1] Aull C.E., Thron W.J., Separation axioms between $T_{0}$ and $T_{1}$, Indag. Math. 24 (1963), 26-37.

[2] Banaschewski, B., Pultr, A., Variants of openness, Appl. Categ. Structures 2 (1994), 331-350.

[3] Davey, B., Priestley, H., Introduction to Lattices and Order, 2nd edition, Cambridge University Press, Cambridge, 2002.

[4] Erné, M., Gehrke M., Pultr, A., Complete congruences on topologies and down-set lattices, to appear in Appl. Categ. Structures.

[5] Hofmann, K.H., Mislove, M., Free objects in the category of completely distributive lattices. In: R.-E. Hoffmann, K.H. Hofmann (Eds.), Continuous Lattices and Applications, Marcel Dekker, Inc., New York - Basel, 1985.

[6] Isbell, J., Atomless parts of spaces, Math. Scand. 31 (1972), 5-32.

[7] Johnstone, P.T., Stone Spaces, Cambridge University Press, Cambridge, 1982.

[8] Johnstone, P.T., Sketches of an Elephant, (Oxford Logic Guides 43\&44), Oxford University Press, Oxford, 2003.

[9] Joyal, A., Tierney, M., An extension of the Galois theory of Grothendieck, Memoirs of the AMS 51 Number 309 (1984).

[10] Kohler, P., Brouwerian semilattices, Trans. of the AMS 268 (1981), 103-126.

[11] Nemitz, W.C., Implicative semi-lattices, Trans. of the AMS 117 (1965), 128-142.

[12] Pultr, A., Frames, Chapter in: Handbook of Algebra, Vol.3, (Ed. by M. Hazewinkel), Elsevier 2003, 791-858.

[13] Pultr, A., Tozzi, A., Separation axioms and frame representation of some topological facts. Appl. Categ. Structures 2 (1994), 107-118.

[14] Simmons, H., The lattice theoretic part of topological separation axioms, Proc. Edinburgh Math. Soc. 21 (1978), 41-48.

[15] Simmons, H., A framework for topology, in: Logic Colloquium 77, Studies in Logic and the Foundations of Mathematics 87, North-Holland, Amsterdam 1978, 239-251.

JoRge PiCADO

Departamento de Matemática, Universidade de Coimbra, 3001-454 Coimbra, PORTUGal E-mail address: picado@mat.uc.pt

Aleš PUltr

Department of Applied Mathematics and iti, MFF, Charles University, Praha, CZeCH REPUBLIC

E-mail address: pultr@kam.ms.mff.cuni.cz

ANNA TOzZI

Dipartimento di Matematica Pura ed Applicata, Università degli Studi di L'Aquila, I 67100 L'Aquila, Via Vetoio, Loc. Coppito, itAly

E-mail address: tozzi@univaq.it 